\title{
Neuronal Activity in Early Visual Areas during Global and Local Processing: A Comment on Heinze, Hinrichs, Scholz, Burchert, and Mangun
}

\author{
Gereon R. Fink \\ University of Düsseldorf \\ John C. Marshall and Peter W. Halligan \\ University of Oxford
}

\author{
R. J. Dolan \\ University College London
}

In a paper published in the 10:4 issue of JOCN, Heinze et al. (Heinze, Hinrichs, Scholz, Burchert, \& Mangun, 1998) studied a directed attention task using hierarchically organized letter Navon stimuli. On the basis of negative PET and ERP findings, they argue that "early sensory inputs are not modulated to gate global vs. local visual information differentially into the two hemispheres." Rather, based on positive ERP findings (an N2 effect) in a divided-attention task, they argue that

"later stages of cortical processing may be asymmetrically organized in the left and right hemispheres and operate in parallel to process global and local aspects of complex stimuli." The authors conclude that their results "support models proposing that spatial frequency analysis is only asymmetric at higher stages of perceptual processing and not at the earliest stages of visual cortical analysis."

In three previous functional-neuroimaging studies of directed-visual attention to complex-hierarchical stimuli of various types (letters made of letters, objects made of objects, and rectangles made of rectangles), we have repeatedly demonstrated hemispheric differences between global and local processing both in early visual processing areas (V2/V3). We also find that higher stages of perceptual processing that activate temporo-parietal cortex are implicated in a divided-attention task using such stimuli (Fink et al., 1996; Fink et al., 1997a). In addition, such hemispheric-differential activations at the early stages of visual processing are influenced by stimulus size (Fink et al., 1996; Fink et al., 1997a), stimulus category (Fink et al., 1997b), spatial frequency and stimulus salience (Fink, Marshall, Halligan, \& Dolan, 1999).

Our PET findings are thus consistent with neuropsychological studies demonstrating a critical role of the temporo-parietal cortex in global/local processing (Ro- bertson \& Lamb, 1991; Robertson, Lamb, \& Knight, 1988), functional-imaging data using fMRI (Martinez et al., 1997) showing hemispheric differences between global and local processing, and other functional-imaging data (using both PET and fMRI) on the attentional gating of early visual processing areas (Corbetta, Miezin, Dobmeyer, Shulman, \& Petersen, 1990). Furthermore, electrophysiological data obtained in monkeys directly demonstrate enhanced neural activity in early visual cortex resulting from changes in attentional set (Motter, 1993; Moran \& Desimone, 1985; Desimone \& Gross, 1979).

The N2 effect observed by Heinze et al. (1998) is consistent with the widely accepted view that the local aspects of hierarchical letter Navon figures are preferentially processed in the left hemisphere while the global aspects of such figures are preferentially processed in the right hemisphere. Leaving aside the problem of the exact localization of ERP sources, it is important to consider whether their negative PET findings (Heinze et al., 1998) can be taken as evidence against our findings of early visual processing differences in similar experiments (Fink et al., 1997a; Fink et al., 1999). The failure of Heinze et al. to observe the differential activations in the early visual processing areas may be due to many factors. For example, their stimuli consisted of only four letters, two of which were locally/globally congruent. Potential conflict between processing levels is thus absent from half the trials in the experiment of Heinze et al. By contrast, we used 12 different letters which were always locally/globally incongruent. The mean RTs obtained by Heinze et al. when their subjects were attending to global and local targets were not significantly different. This lack of a global precedence effect (due perhaps to including congruent stimuli) likewise casts doubt on the appropriateness of the 
particular visual stimuli they used. Proverbio et al. have shown significant differences between congruent and incongruent stimuli using ERP measures (Proverbio, Minniti, \& Zani, 1998).

The design deployed by Heinze et al. includes a socalled "passive" viewing task with the same stimuli used in the experimental trials. This "passive" control task is referred to as "neutral," but is likely to have itself involved implicit global and local processing. If this is so, their statistical analysis involves subtracting one set of implicit global/local tasks from another set of explicit global/local tasks. The likelihood of finding significant differences between the processing types is thus minimized. In another design difference, Heinze et al. displayed each of their stimuli for $100 \mathrm{msec}$. By contrast, stimulus duration in our experiments was $300 \mathrm{msec}$, accordingly allowing more time for differential processing. The imaging technology and analysis applied by Heinze et al. is also weak. Ten subjects were studied, but there were only two replications per condition. Therefore, the statistical analysis of potential differences between locally and globally directed attention was based on $n=40$ observations. For comparison: In the letter Navon study by Fink et al. (1996), 10 subjects were likewise studied but there were six repeats for each globally and locally directed attention condition. The equivalent-statistical analysis was thus based on $n=120$ observations.

On the basis of our functional-imaging data and the results from many neuropsychological and psychophysiological studies, we have argued that neural activity in early visual processing areas does not solely represent the physical attributes of a retinal image. Rather, it is also influenced by attentional modulation via "top-down" processes (i.e., globally/locally directed or divided attention). Other exogenous factors (e.g., changes in spatial frequency, stimulus category or salience) will interact with the neural activations that reflect the processing of either global or local stimulus properties. The negative PET results obtained by Heinze et al. in their most recent study (Heinze et al., 1998) do not argue strongly against such a model (Fink et al., 1999). Furthermore, Proverbio et al. using ERP (Proverbio et al., 1998) have demonstrated an early global interference effect as indexed by the modulation of an early N115 component in a study involving incongruent-hierarchical letters. These electrophysiological data provide further robust evidence for the hypothesis that brain activity in early visual areas can be modulated by an attentional task that is based on distinctions between global and local processing.

\section{Acknowledgments}

Reprint requests should be sent to Gereon R. Fink, Neurologische Klinik, Heinrich-Heine-Universität Düsseldorf, Postfach 1010 07, 40001 Düsseldorf, Germany, or via email: gereon. fink@uni-duesseldorf.de.

\section{REFERENCES}

Corbetta, M., Miezin, F. M., Dobmeyer, S., Shulman, G. L., \& Petersen, S. E. (1990). Attentional modulation of neural processing of shape, color, and velocity in humans. Science, 248, 1556-1559.

Desimone, R., \& Gross, C. G. (1979). Visual areas in the temporal lobe of the macaque. Brain Research, 178, 363380

Fink, G. R., Halligan, P. W., Marshall, J. C., Frith, C. D., Frackowiak, R. S. J., \& Dolan, R. J. (1996). Where in the brain does visual attention select the forest and the trees? Nature, 382, 626-628.

Fink, G. R., Halligan, P. W., Marshall, J. C., Frith, C. D., Frackowiak, R. S. J., \& Dolan, R. J. (1997a). Neural mechanisms involved in the processing of global and local aspects of hierarchically organized visual stimuli. Brain, 120, 17791791.

Fink, G. R., Marshall, J. C., Halligan, P. W., Frith, C. D., Frackowiak, R. S. J., \& Dolan, R. J. (1997b). Hemispheric specialization for global and local processing: The effect of stimulus category. Proceedings of the Royal Society London $B, 264,487-494$.

Fink, G. R., Marshall, J. C., Halligan, P. W., \& Dolan, R. J. (1999). Hemispheric asymmetries in global/local processing are modulated by perceptual salience. Neuropsychologia, 37, 31-40.

Heinze, H. J., Hinrichs, H., Scholz, M., Burchert, W., \& Mangun, G. R. (1998). Neural mechanisms of global and local processing: A combined PET and ERP study. Journal of Cognitive Neuroscience, 10, 485-498.

Martinez, A., Moses, P., Frank, L., Buxton, R., Wong, \& E., Stiles, J. (1997). Hemispheric asymmetries in global and local processing: Evidence from fMRI. NeuroReport, 8, 1685-1689.

Moran, J., \& Desimone, R. (1985). Selective attention gates visual processing in the extrastriate cortex. Science, 229, 782-784.

Motter, B. C. (1993). Focal attention produces spatially selective processing in visual cortical areas V1, V2, and V4 in the presence of competing stimuli. Journal of Neurophysiology, 70, 909-919.

Proverbio, A. J., Minniti, A., \& Zani, A. (1998). Electrophysiological evidence of a perceptual precedence of global vs. local visual information. Cognitive Brain Research, 6, 321334.

Robertson, L. C., \& Lamb, M. R. (1991). Neuropsychological contributions to theories of part/whole organization. Cognitive Psychology, 23, 299-330.

Robertson, L. C., Lamb, M. R., \& Knight, R. T. (1988). Effects of lesions of temporal-parietal junction on perceptual and attentional processing in humans. Journal of Neuroscience, 8 , 3757-3769. 